\title{
Dynamics of Many-Body Photon Bound States in Chiral Waveguide QED
}

\author{
Sahand Mahmoodian $\odot,{ }^{1}$ Giuseppe Calajó $\odot,{ }^{2}$ Darrick E. Chang, ${ }^{2,3}$ Klemens Hammerer, ${ }^{1}$ and Anders S. Sørensen ${ }^{4}$ \\ ${ }^{1}$ Institute for Theoretical Physics, Institute for Gravitational Physics (Albert Einstein Institute), \\ Leibniz University Hannover, Appelstraße 2, 30167 Hannover, Germany \\ ${ }^{2}$ ICFO-Institut de Ciencies Fotoniques, The Barcelona Institute of Science and Technology, \\ 08860 Castelldefels (Barcelona), Spain \\ ${ }^{3}$ ICREA-Institució Catalana de Recerca i Estudis Avançats, 08015 Barcelona, Spain \\ ${ }^{4}$ Center for Hybrid Quantum Networks (Hy-Q), Niels Bohr Institute, University of Copenhagen, \\ Blegdamsvej 17, DK-2100 Copenhagen, Denmark
}

(Received 29 November 2019; revised 7 May 2020; accepted 11 June 2020; published 14 July 2020)

\begin{abstract}
We theoretically study the few- and many-body dynamics of photons in chiral waveguides. In particular, we examine pulse propagation through an ensemble of $N$ two-level systems chirally coupled to a waveguide. We show that the system supports correlated multiphoton bound states, which have a welldefined photon number $n$ and propagate through the system with a group delay scaling as $1 / n^{2}$. This has the interesting consequence that, during propagation, an incident coherent-state pulse breaks up into different bound-state components that can become spatially separated at the output in a sufficiently long system. For sufficiently many photons and sufficiently short systems, we show that linear combinations of $n$-body bound states recover the well-known phenomenon of mean-field solitons in self-induced transparency. Our work thus covers the entire spectrum from few-photon quantum propagation, to genuine quantum many-body (atom and photon) phenomena, and ultimately the quantum-to-classical transition. Finally, we demonstrate that the bound states can undergo elastic scattering with additional photons. Together, our results demonstrate that photon bound states are truly distinct physical objects emerging from the most elementary light-matter interaction between photons and two-level emitters. Our work opens the door to studying quantum many-body physics and soliton physics with photons in chiral waveguide QED.
\end{abstract}

DOI: 10.1103/PhysRevX.10.031011

Subject Areas: Quantum Physics

\section{INTRODUCTION}

Generating quantum many-body states of light remains one of the outstanding challenges of modern quantum optics [1]. Such many-body states of light are of fundamental physical interest as they arise from nonequilibrium systems with strong interactions between light and matter. On the other hand, they also promise to form novel resources for quantum technologies, for example, in quantum-enhanced metrology [2]. The main obstacle in the pursuit of generating such many-body states has been the difficulty in developing one-dimensional systems with a sufficiently strong nonlinear response at the few-photon scale [3]. Recently however, significant progress has been made in creating an ideal light-matter interface between atoms or artificial emitters coupled to a one-dimensional continuum of photons at optical [4-6] and microwave

Published by the American Physical Society under the terms of the Creative Commons Attribution 4.0 International license. Further distribution of this work must maintain attribution to the author(s) and the published article's title, journal citation, and DOI. frequencies [7-9]. Such an interface creates a highly nonlinear medium as photons propagating in a waveguide interact deterministically with atoms. Systems of this kind have thus far been used to propose or demonstrate the generation of states of photons with strong two- or threebody correlations [10-20].

Studies of photon correlations in these systems typically consider steady-state driving, and photon correlations are subsequently measured in relative coordinates. On the other hand, here we show that pulse propagation through twolevel systems (TLSs) strongly coupled to a waveguide leads to very distinct temporal features which reveal the underlying dynamics and has the potential to generate temporally ordered many-body states of light. In particular, we theoretically consider the propagation of pulses of coherent and Fock states of light through a waveguide to which $N$ TLSs are chirally coupled. We show that photons undergo a Wigner delay $[21,22]$ - a delay of the pulse center due the optical excitation transferring to the atom and back to the waveguide - which is dependent on the number of photons. Therefore, incident pulses break up into a state that is temporally ordered by its photon correlations. For example, as shown in Fig. 1(a), an incident coherent field can 
(a)

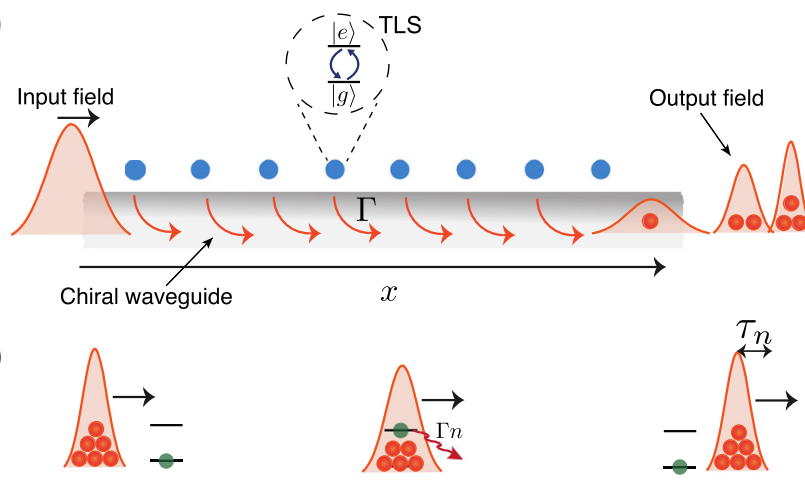

FIG. 1. (a) $N$ two-level atoms (blue circles) are chirally coupled to a waveguide with decay rate $\Gamma$ and driven by an input Gaussian pulse, which can be a coherent or Fock state. The light pulse propagates with a correlation-number-dependent group velocity leading to an output state where one-, two-, and three-photon bound states are spatially separated. (b) Schematic of the bound-states propagation. When an $n$-photon bound state is scattered by an atom, it reemits the absorbed photon with a stimulated emission rate $\Gamma n$, coinciding with the inverse of its width. Since only a single photon out of $n$ is delayed by an amount $4 /(\Gamma n)$, the pulse preserves its shape but is delayed by $\tau_{n}=4 /\left(\Gamma n^{2}\right)$.

produce a pulse with three-photon correlations followed by two-photon correlations states followed by uncorrelated photons. The underlying physics that causes this time delay is examined by considering the many-body photonic scattering eigenstates of TLSs chirally coupled to a waveguide.

Of central importance are the class of bound eigenstates, where two or more photons propagate together. We show that the photon bound states propagate past each atom with a photon-number-dependent delay of $\tau_{n}=4 /\left(n^{2} \Gamma\right)$, where $\Gamma$ is the decay rate into the waveguide, as can be understood in terms of absorption and stimulated emission of a photon as shown in Fig. 1(b). Since the interaction is chiral, the delay is also proportional to the number of emitters $N$ in the waveguide. We also show that the pulse distortion encountered by an $n$-photon bound state scales as $n^{-6}$. Pulses of higher-number bound states therefore propagate with negligible distortion, i.e., much like a soliton. Moving beyond the few-photon-few-atom limit, we obtain a simple description of photon propagation with a mesoscopic number of photons $n$ but a large number of atoms $N \gg 1$. Here, the number-dependent group delay breaks the input pulse apart and produces a many-body ordered state of light. Finally, we show that the system approaches the classical limit for $n \gg 1$ and $n \gg N$, where our full quantum description captures the quantum-to-classical transition and reproduces the mean-field results of soliton propagation in self-induced transparency.

The effect we discuss here has features similar to vacuum-induced transparency (VIT) where photons undergo a photon-number-dependent delay after interacting with atoms coupled to a cavity [23,24]. A key difference is that the delay in VIT mainly depends on the total photon number inside the entire system, and not on the details of the pulse shape. The nonlinear effect we consider is spatially localized to the individual atoms and occurs for the simplest possible configuration of TLSs. This leads to a different spatiotemporal behavior which we evaluate in a full multimode theory of the dynamics. Our theory features a full quantum many-body treatment of the system, where the photon time delay is examined by considering the many-body photonic scattering eigenstates.

We also point out that in mean-field theories, solitons are known to be highly stable objects which are unaffected by external perturbations. Here we show that similar properties exist for few-photon bound states. We outline how one can conduct scattering experiments between photon bound states and individual photons. In the considered scattering experiments, the bound state is deflected by the interaction but is otherwise unperturbed by it. Together, the results we obtain here demonstrate that the bound states should be considered as truly distinct physical entities emerging from the underlying light-matter interaction between photons and two-level emitters.

This manuscript is arranged as follows: In Sec. II, we introduce the model for chiral waveguide QED (WQED) and outline various theoretical approaches for computing the dynamics through Sec. II A mean-field theory, Sec. II B the photon-scattering eigenstates, and Sec. II C the matrixproduct states (MPSs). In Sec. III, we compute how the input pulse propagates through the medium and compute the representation in terms of photon bound states. This is followed by Sec. IV, which shows that the many-body photon bound states can be used to construct the mean-field soliton solutions obtained in self-induced transparency. In Sec. V, we show that photon bound states can undergo elastic scattering with individual photons modifying the delay of the bound state but otherwise leaving it unaltered, much like classical solitons. In Sec. VI, we show that few-photon bound-state propagation can potentially be observed in state-of-the-art experiments with a few emitters, and we discuss potential future applications. Finally, we conclude in Sec. VII.

\section{MODEL}

We consider a system of $N$ TLSs chirally coupled to a linearly dispersive one-dimensional bath of photons. Here, chiral coupling means that the TLSs couple only to rightpropagating photons. The Hamiltonian for this system $(\hbar=1)$ is

$$
\hat{H}=-i \int d x \hat{a}^{\dagger}(x) \partial_{x} \hat{a}(x)+\sqrt{\Gamma} \sum_{i=1}^{N}\left[\hat{\sigma}_{i}^{-} \hat{a}^{\dagger}\left(x_{i}\right)+\hat{\sigma}_{i}^{+} \hat{a}\left(x_{i}\right)\right]
$$


where all integrals are over $\Re, \hat{\sigma}_{i}^{ \pm}$are Pauli operators for the $i$ th TLS, $\hat{a}(x)\left[\hat{a}^{\dagger}(x)\right]$ is a photon annihilation (creation) operator at position $x$, the group velocity is set to unity $v_{g}=1$, and the energy is renormalized to that of the TLS. In the limit of ideal chiral coupling, the system dynamics are not influenced by the positions of the TLSs $x_{i}$. In this Hamiltonian, the first term captures the free propagation of photons in the waveguide with linear dispersion, while the next terms take into account the interaction between the photons and the emitters. In the absence of the interaction terms, the photonic eigenstates of the Hamiltonian are plane waves with wave vector $k$ and frequency $\omega=k$. Equation (1) constitutes the typical scenario for chiral WQED [25]. In this manuscript, we are interested in describing the propagation of a multiphoton input field through the system. This goal can be achieved by exactly computing the scattering eigenstates of Hamiltonian (1), as we illustrate in Sec. II B.

In addition to computing the eigenstates of Eq. (1), we also introduce an equivalent formulation that is well suited for using the MPS technique that we introduce later in Sec. II C. This approach is based on the observation that the transmitted field depends directly on the emitters' evolution. This can be seen by formally integrating the Heisenberg equation for the field operator $\hat{a}(x, t)$, that provides, within the Born-Markov approximation, the following generalized input-output relation for the transmitted field [26,27]

$$
\hat{a}_{\text {out }}(t)=\mathcal{E}_{\text {in }}(t)+i \sum_{j} \sqrt{\Gamma} \hat{\sigma}_{j}^{-}(t),
$$

where we define $\hat{a}_{\text {out }}(t)=\hat{a}\left(x_{N}^{+}, t\right)$ as the output field measured right after the last atom. Note that, within this approach, we assume the input field $\mathcal{E}_{\text {in }}(t)$ to be a classical coherent field on resonance with the atomic transitions. With these assumptions, the emitter dynamics driven by the input field is known to be described by a purely dissipative chiral master equation (ME) of the form [28,29] (see the Supplemental Material [30] for more information):

$$
\dot{\rho}=-i\left(H_{\mathrm{eff}} \rho-\rho H_{\mathrm{eff}}^{\dagger}\right)+\Gamma \sum_{i j} \hat{\sigma}_{i}^{-} \rho \hat{\sigma}_{j}^{+} .
$$

Here,

$$
H_{\mathrm{eff}}=-i \frac{\Gamma}{2} \sum_{j} \hat{\sigma}_{j}^{+} \hat{\sigma}_{j}^{-}+H_{\mathrm{drive}}-i \Gamma \sum_{l>j} \hat{\sigma}_{l}^{+} \hat{\sigma}_{j}^{-}
$$

is the effective Hamiltonian, which provides the nonHermitian collective evolution of the emitters, while the term $\mathrm{H}_{\text {drive }}=\sum_{j} \sqrt{\Gamma}\left[\mathcal{E}_{\text {in }}(\mathrm{t}) \hat{\sigma}_{j}^{+}+\right.$H.c. $]$gives the coupling of the emitters to the input field.

The combination of Eqs. (2) and (3) provides a full description of the photon propagation through the chiral medium. In particular, the spin dynamics can be efficiently solved by making use of a MPS ansatz [31,32], as recently described in Ref. [27]. As we show in the following, this approach allows us to fully explore the limit of many photons and large atomic arrays, a scenario that is challenging to simulate with standard numerical techniques.

\section{A. Mean-field theory and self-induced transparency}

Before considering the full many-body dynamics of the Hamiltonian (1), we consider the system within the mean-field limit. We present this mean-field limit to contrast its predictions with the full many-body theory that we present below.

The first treatment of Eq. (1) within mean-field theory dates back to the work on self-induced transparency (SIT) [33-35]. In these early experiments, gasses of two-level atoms were excited by short intense laser pulses. Although the atoms are not ideally coupled to a single-waveguide mode in such systems, the laser pulses are sufficiently short so that decay channels to modes other than the laser mode can be neglected. Furthermore, both the weak coupling of the atoms to this mode and the high intensity of the laser means that one can consider the atoms as a spin continuum under the mean-field approximation where quantum correlations between the atoms and the light field can be neglected. Under these approximations, the equations of motion give the SIT equations

$$
\begin{aligned}
{\left[\frac{\partial}{\partial t}+\frac{\partial}{\partial x}\right] a(x, t) } & =-i \sqrt{\Gamma} \sigma_{-}(x, t), \\
\frac{\partial}{\partial t} \sigma_{-}(x, t) & =i \sqrt{\Gamma} \sigma_{z}(x, t) a(x, t), \\
\frac{\partial}{\partial t} \sigma_{z}(x, t) & =4 \sqrt{\Gamma} \operatorname{Im}\left[a(x, t) \sigma_{-}^{*}(x, t)\right] .
\end{aligned}
$$

Here, $a(x, t)=\langle\hat{a}(x, t)\rangle, \hat{\sigma}_{-/ z}(x, t)=\sum_{i} \hat{\sigma}_{i}^{-/ z}(t) \delta\left(x-x_{i}\right)$, and $\sigma_{-}(x, t)=\left\langle\hat{\sigma}_{-}(x, t)\right\rangle, \quad \sigma_{z}(x, t)=\left\langle\hat{\sigma}_{z}(x, t)\right\rangle$ are the expectation values of the spin operators in the continuum limit. These nonlinear equations of motion have SIT soliton solutions. Following the treatment in Ref. [35], for a resonant pulse the field can be taken to be real, and one can map the equations of motion onto a nonlinear pendulum equation that can be solved exactly. This treatment leads to the fundamental soliton solution for the field

$$
a(x, t)=\frac{\sqrt{\Gamma} \bar{n}}{2} \operatorname{sech}\left[\frac{\Gamma \bar{n}}{2}\left(\frac{x}{V^{\prime}}-t\right)\right],
$$

where $\bar{n}$ is the number of photons in the field, (or more precisely, the total energy in the original SIT work). The pulse velocity within the medium in the laboratory frame is $V^{\prime}=\bar{n}^{2} \Gamma /\left(\bar{n}^{2} \Gamma+4 \nu\right)$, where $\nu$ is the gas density (see the Supplemental Material [30] for details). Transforming to a frame comoving with the pulse in the absence of emitters, 
i.e., at velocity $v_{g}=1$, gives the relative velocity in the backwards direction $V=4 \nu /\left(\bar{n}^{2} \Gamma+4 \nu\right)$. This value for the relative velocity corresponds to each emitter imparting a delay of $\tau_{\bar{n}}=4 /\left(\bar{n}^{2} \Gamma\right)$ on the pulse.

An important feature of the solitonic solution (6) is that the integrated Rabi frequency $\Omega=2 \sqrt{\Gamma} \int d t a(x, t)$, which is proportional to the area under the pulse, is fixed by the relationship between the pulse amplitude and pulse width, and always evaluates to be $2 \pi$. This corresponds to a full Rabi cycle of complete excitation and subsequent deexcitation. The SIT soliton therefore can be physically interpreted as a rapid excitation and deexcitation of the atoms, which suppresses spontaneous emission of the excited state and thus makes the medium transparent.

Inspired by the apparent dependence of the velocity on photon number, it is interesting to ask whether this property extends to the few-photon limit, thus enabling, e.g., photonnumber separation at the output. A full quantum treatment is necessary to answer this question, which we turn to in the following sections.

\section{B. Many-body scattering eigenstates}

In contrast to the mean-field treatment, we now consider the full many-body eigenstates. Since the Hamiltonian (1) preserves the combined number of atomic and photonic excitations, eigenstates with different numbers of excitations decouple. By computing the eigenstates in the oneand two-excitation subspaces, one can generalize the result to an arbitrary number of excitations. This technique is often referred to as Bethe's ansatz [36] and is used to diagonalize a class of one-dimensional many-body Hamiltonians [37]. In particular, it has previously been used to diagonalize the Hamiltonian in Eq. (1) [38]. Since we are interested in the state that emerges after interaction with the TLSs, we are interested in the scattering eigenstates. These are photon eigenstates that interact with the TLSs and emerge unchanged apart from an overall transmission coefficient. The $n$-body scattering eigenstates have the form

$$
\begin{aligned}
\left|S_{\mathbf{k}}\right\rangle_{n}= & C_{\mathbf{k}, n, S} \frac{1}{\sqrt{n !}} \int d^{n} \mathbf{x} \hat{\mathbf{a}}^{\dagger}(\mathbf{x})|0\rangle \\
& \times \prod_{l<m}^{n}\left[k_{l}-k_{m}+i \Gamma \operatorname{sgn}\left(x_{l}-x_{m}\right)\right] \prod_{j=1}^{n} e^{i k_{j} x_{j}}+\leftrightarrow,
\end{aligned}
$$

where $C_{\mathbf{k}, n, S}$ is a normalization constant which varies with wave vector $\mathbf{k}$, excitation number $n$, and the type of eigenstates $S$, where $S$ labels different states as explained below; $\hat{\mathbf{a}}^{\dagger}(\mathbf{x})=\hat{a}^{\dagger}\left(x_{1}\right) \hat{a}^{\dagger}\left(x_{2}\right), \ldots, \hat{a}^{\dagger}\left(x_{n}\right) ; d^{n} \mathbf{x}=$ $d x_{1} d x_{2}, \ldots, d x_{n}$, and $\leftrightarrow$ indicates summing over all $n$ ! permutations of $x_{i}$ to symmetrize the wave function. The energy of the eigenstates is $E=\sum_{i}^{n} k_{i}$. Upon scattering off all $N$ emitters, the eigenstates are multiplied by the eigenvalue $t_{\mathbf{k}}^{N}=\prod_{j=1}^{n} t_{k_{j}}^{N}$, where $t_{k}=(k-i \Gamma / 2) /$ $(k+i \Gamma / 2)$. Since, by assumption, the system does not contain any dissipation and is chiral, all the transmission coefficients have $\left|t_{\mathbf{k}}\right|=1$. This means that the transmission coefficients simply multiply the eigenstate by a phase. For example, a single photon on resonance undergoes a $\pi$-phase shift with $t_{0}=-1$. Importantly, the phase that is imparted on the eigenstate varies with k; i.e., the TLSs introduce dispersion to the system. As we soon show, different types of eigenstates also accumulate different phases. We note that the output states are described using the position coordinates $\mathbf{x}$. This is equivalent to using the time variable $-t$ in Eq. (2) as we set the group velocity to unity.

In addition to different eigenstates for different values of $n$, there are also different possible types of eigenstates within different excitation-number manifolds. In the singleexcitation subspace, there is only one type of eigenstate and it is characterized by a real wave number $k$ and the eigenstate is fully extended in space. For $n=2$, the wave numbers $k_{1}$ and $k_{2}$ can either both be real which gives rise to a fully extended solution of the form (7). They can also assume complex values $k_{1}=E / 2+i \Gamma / 2$ and $k_{2}=E / 2-$ $i \Gamma / 2$ which are called strings [37]. These values give additional valid solutions. Since the wave vectors can form complex conjugate pairs, the eigenstates become localized in a relative coordinate while being fully extended within the center-of-mass coordinate. This localization is associated with the formation of photon bound states that manifest in the bunching of two photons which travel together during their propagation $[39,40]$.

For $n>2$, the $m$-body string $(m \leq n)$ has the wave vector $k_{j}=K / m-i[m+1-2 j] \Gamma / 2$ with $j=1,2, \ldots, m$ where $K$ is the total energy of the $m$ string. In total, the $n$-photon manifold has $p(n)$ string combinations where $p(n)$ is the number of partitions of $n$. For example, for three photons, there is a completely extended scattering eigenstate, a completely bound eigenstate, and a hybrid state with two bound photons and one extended photon. The different string combinations give the different types of scattering eigenstates $S$ by substituting the complex wave vectors into Eq. (7). The transmission coefficient is also obtained by substituting the complex wave vectors into the expression for $t_{\mathbf{k}}$.

For $n$ photons, the $n$-string state is a fully localized bound state with energy $E$,

$$
\left|B_{E}\right\rangle_{n}=\frac{C_{n, B}}{\sqrt{n !}} \int d^{n} \mathbf{x} \hat{\mathbf{a}}^{\dagger}(\mathbf{x})|0\rangle e^{i(E / n) \sum_{j} x_{j}-(\Gamma / 2) \sum_{i<j}\left|x_{i}-x_{j}\right|},
$$

where $C_{n, B}=\sqrt{\Gamma^{n-1}(n-1) ! /(2 \pi n)}$. The transmission coefficient for the $n$-photon bound state is then 


$$
t_{E, n}=\frac{E-i \Gamma n^{2} / 2}{E+i \Gamma n^{2} / 2} .
$$

Importantly, the phase of the transmission coefficient varies with $n$; i.e., the system has a photon-number-dependent dispersion.

With all the eigenstates at hand, the scattering matrix for interacting with all $N$ emitters in the $n$-photon manifold can be formally written as

$$
\hat{S}_{n}^{N}=\sum_{S} \sum_{\mathbf{k}} t_{\mathbf{k}}^{N}\left|S_{\mathbf{k}}\right\rangle_{n n}\left\langle S_{\mathbf{k}}\right|,
$$

where the sum over $S$ is a sum over the different string combinations of the $n$-photon manifold. We note that the eigenstates are orthogonal; thus, the scattering matrix for $N$ emitters simply requires taking the eigenvalue to the $N$ th power. Since the number of string combinations increases as $p(n)$, the number of terms in the sum increases exponentially for large $n[38,41]$. In this manuscript, we compute the full output states for up to $n=3$ using this formalism. We note that a formalism exists where one does not have to sum over string combinations [42]. Nevertheless, the form used here is particularly insightful as it gives direct access to the number-dependent transmission coefficient which, as we show, plays a central role in understanding the many-body pulse propagation.

\section{MPS ansatz}

In order to study the dynamics for stronger input pulses $(n>3)$ than the one computed with the $S$-matrix formalism, we solve Eqs. (2) and (3) using a MPS ansatz. Specifically, the system evolution can be solved either by directly solving the ME (3) [43] [method used for Figs. 2(d) and 3] or by using a quantum trajectories algorithm where the state of the system evolves under the effective Hamiltonian (4) and stochastically experiences quantum jumps [27] [method used for Fig. 2(f)]. In both cases, a MPS representation is applied either to the quantum state or to a vectorized form of the density matrix. Here for simplicity, we limit the discussion to the former, while the latter is discussed in the Supplemental Material [30].

The MPS ansatz consists of reshaping the generic quantum state $|\phi\rangle=\sum_{i_{1}, \ldots, i_{N}} \psi_{i_{1}, i_{2}, \ldots, i_{N}}\left|i_{1}, i_{2}, \ldots, i_{N}\right\rangle$ (with $\left.i_{j} \in\{g, e\}\right)$ into a matrix-product state of the form:

$$
|\phi\rangle=\sum_{i_{1}, \ldots, i_{N}} A_{i_{1}} A_{i_{2}}, \ldots, A_{i_{N}}\left|i_{1}, i_{2}, \ldots, i_{N}\right\rangle,
$$

where, for each specific set of physical indices $\left\{i_{1}, i_{2}, \ldots, i_{N}\right\}$, the product of the $A_{i_{j}}$ matrices gives back the state coefficient $\psi_{i_{1}, i_{2}, \ldots, i_{N}}$. Each matrix $A_{i_{j}}$ has dimension $D_{j-1} \times D_{j}$ known as the bond dimension, and finite-edge boundary conditions are assumed by imposing
$D_{1}=1$ and $D_{N}=1$. The bond dimension reflects the entanglement entropy. For instance, if $D_{j}=1$ for all $j$, the matrices $A_{i_{j}}$ are scalars and the state (11) reduces to a product state with no entanglement. For arbitrary states, the bond dimension grows exponentially with the number of particles. The advantage of the MPS ansatz is that, in many physical scenarios as the one considered here, the entanglement grows slowly with the system size allowing an efficient description of the state in terms of a smaller bond dimension [32]. An important figure of merit of the efficiency of the MPS ansatz is given by $D_{\max }$, the maximum bond dimension that is needed to faithfully represent the system during the entire time evolution (see the Supplemental Material [30] for more information). We make use of this quantity in Sec. IV to quantify the amount of many-body correlations present in the system.

\section{MANY-BODY PULSE PROPAGATION}

We are interested in studying multiphoton propagation through the chirally coupled array. Here, we consider coherent and Fock input states with mode creation operator $\hat{a}_{\text {in }}^{\dagger}=\int d x \mathcal{E}(x) \hat{a}^{\dagger}(x)$, and we evaluate the transmitted field with the two methods described in the previous section. In particular, for the exact solution we compute the transmitted photon state using the eigenstates for up to three photons, while for higher excitations we make use of the MPS ansatz. In Fig. 2, we consider the propagation of a Gaussian photonic mode with amplitude $\mathcal{E}(x)=$ $e^{i k_{0} x-x^{2} /\left(2 \sigma^{2}\right)} /\left(\sqrt{\sigma} \pi^{1 / 4}\right)$ where, throughout the manuscript, resonant pulses are considered $k_{0}=0$.

Figures 2(a)-2(c) show the power $P(x)=\left\langle\hat{a}^{\dagger}(x) \hat{a}(x)\right\rangle$ for one-, two-, and three-photon Fock states after propagating through $N=20$ TLSs. Here, $x=0$ is chosen to be the reference frame of the pulse propagating in the absence of emitters. A pulse width of $\Gamma \sigma=3 \sqrt{2}$ is chosen to have appreciable overlap with all the different types of scattering eigenstates while remaining sufficiently narrow to observe the photon-number-dependent velocities. The magnitude of the overlap of an input Gaussian pulse with two- and threephoton bound states versus Gaussian pulse width $\sigma$ is shown in the Supplemental Material [30]. In Fig. 2(b), we see that the two-photon bound state comes out earlier than the extended state. The two-photon bound state thus clearly propagates with a faster velocity than the extended state. The bound state also undergoes significantly less broadening and distortion. For the three-photon transport in Fig. 2(c), again the extended state is distorted and delayed, while the three-photon bound state has significantly less distortion and delay. In the three-photon manifold, there is also a string combination that forms a hybrid state where one photon is completely extended while the other two are bound. The evolution of this state is determined by the individual components of the state: The two bound photons propagate in a similar manner to the two-photon bound 

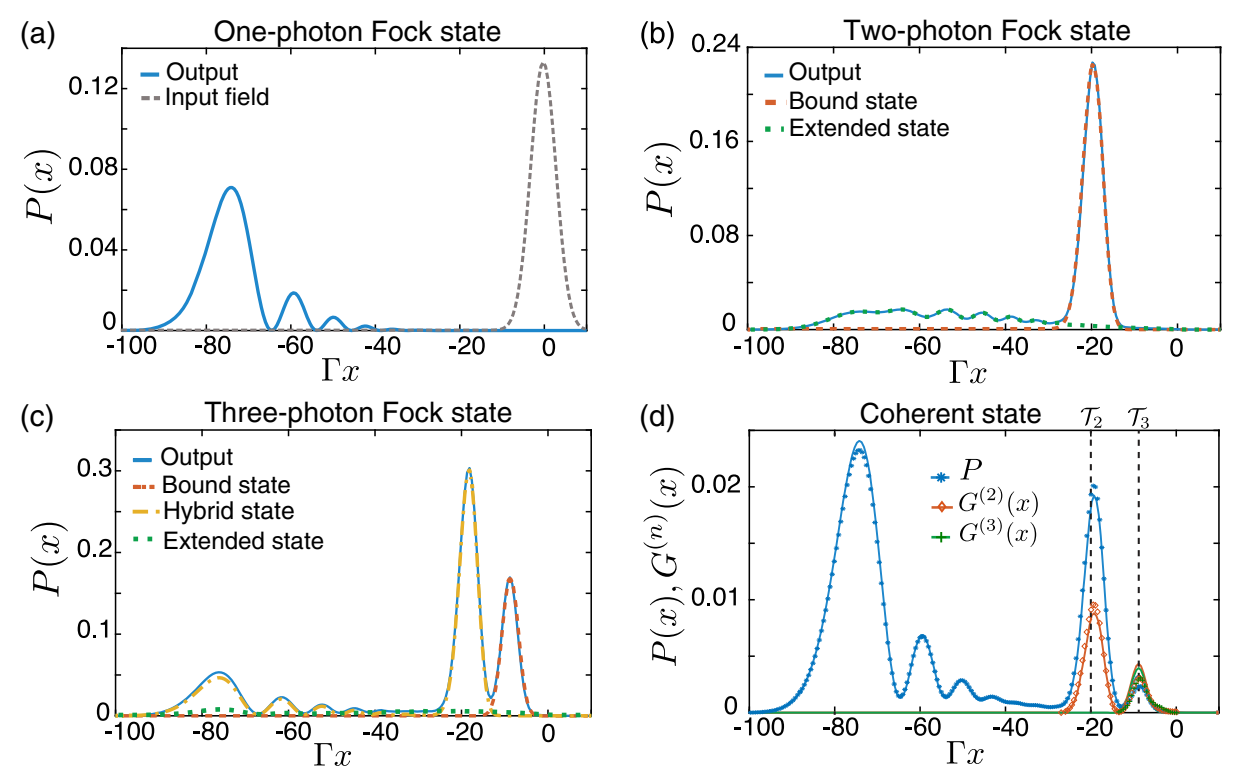

(e)
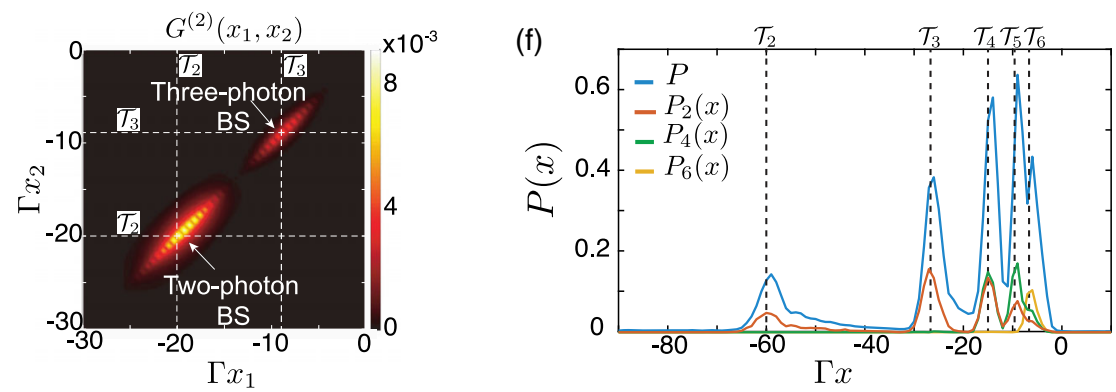

FIG. 2. Transmitted power $P(x)$ (solid curves) for (a) one- (b) two-, and (c) three-photon transport through $N=20$ TLSs for a resonant pulse with $\Gamma \sigma=3 \sqrt{2}$ computed using the photon-scattering theory. In (b) and (c), dashed lines show the contributions from the different eigenstates. The input mode in all panels is the Gaussian curve shown in (a). (d) Transmitted power and correlation functions for a coherent state with $\bar{n}=0.5$. Solid lines show photon-scattering results truncating at three photons, while marker points show MPS calculations performed by solving the ME with a Runge-Kutta algorithm and using maximum bond dimension $D_{\max }=150$. (e) Secondorder correlation function $G^{(2)}\left(x_{1}, x_{2}\right)$ for the transmitted coherent state computed using photon-scattering theory. The two- and threephoton bound-state contributions appear as peaks that are localized in the relative coordinate. (f) MPS calculation for input coherent state with $\bar{n}=8, N=60$ emitters, and $\Gamma \sigma=2 \sqrt{2}$. Dashed vertical lines show $N$ times the Wigner delay $\mathcal{T}_{n}=N \tau_{n}=4 N /\left(\Gamma n^{2}\right)$. With $P_{n}(x)$, we indicate the contribution to the power coming from the emission of $n$ photons within the space bin $\Delta x=1$. To perform this calculation, we use a quantum trajectories MPS algorithm fixing the maximum bond dimension to $D_{\max }=40$.

state in Fig. 2(b), while the extended photon propagates like a single photon. This separation of the propagation of the bound and extended parts of this state can be shown explicitly in the long pulse limit $\sigma \Gamma \gg 1$ (see the Supplemental Material [30]). This is significant because it implies that in order to understand the pulse evolution, one does not have to understand the behavior of all the different string combinations $S$. Rather, one can focus on simply understanding the behavior of the bound states. On the other hand, for short pulses this separation is not completely true because one needs to include the effect of interactions between the components; see Sec. V.

\section{A. Evolution of bound states}

To better quantify the difference in propagation observed above, let us compute the pulse evolution in the center-of-mass coordinate. This can be done by using the form of the $n$-photon bound state and its transmission coefficient given in Eqs. (8) and (9). Within the $n$-photon manifold, the projection of an input Gaussian state on the bound state is ${ }_{n}\left\langle B_{E}\right|$ in $\rangle=c_{n} e^{-\left(E-n k_{0}\right)^{2} \sigma^{2} /(2 n)}$, where $c_{n}$ is a constant in $E$. Here, it is convenient to use Jacobi coordinates $x_{c}=\sum_{j}^{n} x_{j} / n, x_{i}^{J}=\sum_{j=1}^{i} x_{j} / i-x_{i+1}$, where $i \in\{1,2, \ldots, n-1\}$. The resulting bound-state contribution is then

$$
\begin{aligned}
\mid \text { out }\rangle_{n, \text { bound }}= & \frac{c_{n}}{\sqrt{n !}} \int d^{n} \mathbf{x} \hat{\mathbf{a}}^{\dagger}(\mathbf{x})|0\rangle e^{-(\Gamma / 2) g\left(\mathbf{x}^{J}\right)} \\
& \times \int d E t_{E, n}^{N} e^{i E x_{c}-E^{2} \sigma^{2} /(2 n)},
\end{aligned}
$$


where $g\left(\mathbf{x}^{J}\right)$ is the exponent $\sum_{i<j}\left|x_{i}-x_{j}\right|$ written in Jacobi coordinates. The center-of-mass evolution is clearly determined by the second integral. Equation (12) now has the standard form of Gaussian pulse propagation through a linear dispersive medium. Defining $t_{E, n}^{N} \equiv e^{i N \phi(E)}$, the first to third derivatives of $\phi(E)$ give, respectively, the delay, broadening, and distortion that the pulse undergoes per emitter. The delay per emitter is $\tau_{n}\left(k_{0}\right)=$ $\Gamma /\left(k_{0}^{2}+n^{2} \Gamma^{2} / 4\right)$, which is largest for a resonant pulse $\left(k_{0}=0\right)$, where

$$
\tau_{n}=\frac{4}{n^{2} \Gamma}
$$

This gives the Wigner delay imparted on an $n$-photon bound state by a single emitter in WQED: The photons propagate with a number-dependent velocity.

By taking higher-order derivatives, we also compute the pulse broadening $b\left(k_{0}\right)=-32 k_{0} \Gamma /\left[n\left(4 k_{0}^{2}+n^{2} \Gamma^{2}\right)^{2}\right]$, which is zero on resonance. The third-order pulse distortion term on resonance is $d=-32 /\left(n^{6} \Gamma^{3}\right)$. The pulse distortion is thus drastically reduced for higher-order bound states. This indicates that many-photon bound states suffer negligible pulse distortion while propagating through the array of nonlinear and dispersive atoms.

In order to verify that indeed the physics of the bound states dominates the wave-packet evolution, we also compute the evolution of a coherent input pulse as shown in Fig. 2(d). Here, the pulse width $\Gamma \sigma=3 \sqrt{2}$ is the same as in Figs. 2(a)-2(c), while the average photon number in the pulse is $\bar{n}=0.5$. We compute the output both by truncating the coherent state to three photons, and solving exactly using Eq. (10), or by solving Eqs. (2) and (3) with the MPS algorithm. The evolution of the bound states is seen in the position of the peaks of the power distribution $\left\langle\hat{a}^{\dagger}(x) \hat{a}(x)\right\rangle$ as well as in the difference between the power and the $m$ thorder correlation functions $G^{(m)}(x)=\left\langle\left[\hat{a}^{\dagger}(x)\right]^{m}[\hat{a}(x)]^{m}\right\rangle$.

The localized nature of the bound states in the relative coordinates is shown in Fig. 2(e) where we show the two-point second-order correlation function $G^{(2)}\left(x_{1}, x_{2}\right)=$ $\left\langle\hat{a}^{\dagger}\left(x_{2}\right) \hat{a}^{\dagger}\left(x_{1}\right) \hat{a}\left(x_{1}\right) \hat{a}\left(x_{2}\right)\right\rangle$. Here the photons tend to localize around the diagonal at small values of the relative coordinate $x_{1}-x_{2}$, while they are delocalized about the center-of-mass coordinate $\left(x_{1}+x_{2}\right) / 2$. This reflects that the photons are tightly bound together in the bound state, but the bound state itself is free to propagate. While exact analytical calculations become infeasible for $\bar{n} \gg 1$, the validity of our arguments and the importance of the bound states can still be seen in MPS simulations. For example, in Fig. 2(f), we calculate the transmitted power for an input coherent state $\bar{n}=8$ and $N=60$. Here, for this particular system length, the photon-number-dependent Wigner delay clearly manifests itself as separate peaks for up to six-photon bound states.
The low-distortion propagation of the bound states can be intuitively explained by returning to the simple schematic shown in Fig. 1(b). When a multiphoton bound state propagates through the atomic array, one of the photons in the wave packet can be absorbed and reemitted by the atom. This process occurs on a time scale ruled by the inverse of the photon-number-dependent stimulated emission rate that coincides with the bound-state packet width $\Delta t_{n} \sim 1 /(\Gamma n)$ allowing the pulse to preserve its shape. This continuous absorption and reemission of photons during the boundstate propagation leads to a time delay of one out of $n$ photons by an amount $4 /(\Gamma n)$, leading to the group delay in Eq. (13).

\section{B. Influence of imperfections}

We show that the hallmark of many-body photon propagation through an ensemble of quantum emitters in chiral WQED is the number-dependent velocity of the photon bound states. Here we analyze the influence of imperfections such as losses, imperfect chirality, and inhomogeneous broadening on this propagation. We first consider the influence of losses, where each emitter couples to an additional decay channel out of the waveguide with a rate $\Gamma_{0}$. It is possible to obtain an analytic criterion for when these losses can be neglected. The form of the transmission coefficient of the bound state can be obtained in the presence of loss by mapping the total energy to a complex energy using the replacement $E \rightarrow E+i n \Gamma_{0} / 2$. The reduction in probability of the output state then implies that the state can undergo one or more quantum jumps. If the probability remains close to unity, the output state is only weakly affected. Defining the efficiency or $\beta$ factor as $\beta=\Gamma / \Gamma_{\text {tot }}$, where $\Gamma_{\text {tot }}=\Gamma+\Gamma_{0}$, the transmission coefficient (9) in the presence of loss is

$$
t_{E, n}=\frac{E+i n \Gamma_{\text {tot }}[1-\beta(1+n)] / 2}{E+i n \Gamma_{\text {tot }}[1-\beta(1-n)] / 2} .
$$

After scattering off all $N$ emitters the magnitude of the resulting state is $\left|t_{E, n}\right|^{N}$. For small imperfections $1-\beta \ll 1$, this gives $\left|t_{E, n}\right|^{N}=1-2 N(1-\beta) / n+$ $\mathcal{O}\left((1-\beta)^{2}\right)$, where the notation $\mathcal{O}(M)$ indicates a term of order $M$ and higher. This means that a sufficient condition for neglecting losses is $N(1-\beta) / n \ll 1$. This implies that losses have a reduced influence on higherorder bound states. If this condition is not met, there is a sizable probability that one or more of the photons in a photon bound state is lost.

If a photon is lost at one point along the ensemble, the remaining photons propagate through the rest of the atoms with a different effective group velocity and dispersion. In addition to a reduced amplitude, the fact that the remaining transmitted photons have effectively propagated with a mix of velocities and dispersions causes the peaks associated 
with the different bound states in Fig. 2 to broaden and eventually overlap (see the Supplemental Material [30]).

In addition to photon loss, we note that a nonzero value of $\Gamma_{0} \neq 0$ also affects the delay $\tau_{n}$, which is also computed analytically, $\tau_{n}=4 \beta^{3} /\left\{\Gamma\left[\beta\left(2+\beta\left(n^{2}-1\right)\right)-1\right]\right\}=$ $4 /\left(\Gamma n^{2}\right)-4(1-\beta) /\left(\Gamma n^{2}\right)+\mathcal{O}\left[(1-\beta)^{2}\right]$. Values where $\tau_{n}$ diverges occur when $\left|t_{E, n}\right|$ approaches zero; i.e., no light is transmitted.

In the limit of large losses, the photon bound states suffer exponential damping. The transmission of a steady-state input field was considered for up to two photons in Ref. [19], where it was shown that the output shows strong photon bunching. This bunching, however, arises from the extended states and does not reflect the bound-state dynamics. Even with finite-pulse durations, it is likely that the bound-state dynamics will be hard to discern in this limit.

The introduction of imperfect chirality also influences the nature of pulse propagation through the ensemble. Imperfect chirality occurs when, in addition to coupling to the forwardpropagating mode, each atom also couples to the backwardpropagating mode with a rate $\Gamma_{L}$. The influence of imperfect chirality cannot be considered analytically in a straightforward manner. We perform numerical MPS calculations (see the Supplemental Material [30]) for $N=20$ emitters. When $\Gamma_{L}=0.05 \Gamma$, the shape of the output state remains qualitatively unchanged. However, when $\Gamma_{L}=0.2 \Gamma$ the output state is completely distorted. These computations indicate that the effect observed in the ideal chiral case is robust to imperfect chirality, provided that the imperfections are not too large.

We also consider the influence of inhomogeneous broadening in the two-level systems. The two-level atoms are considered to have a normally distributed resonance frequency with dimensionless standard deviation $\varsigma / \Gamma$. This inhomogeneous broadening affects the transmission coefficient of the bound states, which most notably affects the pulse delay. An expression for the mean pulse delay can be computed analytically, and for small broadening, gives to leading order

$$
\left\langle\tau_{n}\right\rangle=\tau_{n}\left[1-\frac{4 \varsigma^{2}}{n^{2} \Gamma^{2}}+\mathcal{O}\left(\frac{\varsigma^{4}}{\Gamma^{4}}\right)\right] .
$$

The reduction in the delay imparted by each emitter therefore scales quadratically in $\varsigma /(n \Gamma)$. Inhomogeneous broadening therefore has a reduced impact on higher-order bound states provided that the broadening is limited $\lesssim \Gamma$. We note that the influence of inhomogeneous broadening can be compensated by introducing more emitters.

\section{CONNECTION TO THE SIT SOLITON}

In the previous sections, we show how the Hamiltonian (1) leads to the SIT solitonic solutions in the mean-field limit, and that the full quantum-mechanical treatment of this Hamiltonian predicts correlation-ordered photon propagation. In this section, we aim to bridge the gap between these two regimes: First we show that indeed the many-body theory reduces to the mean-field result in the limit of large photon number. Second, we derive the quantum corrections to the mean-field results which become relevant when both the number of photons $n$ and the number of emitters $N$ are large. Finally, we push the numerical simulations to the many-photon limit to verify the analytical predictions.

Let us consider a wave packet composed of a linear combination of many-body bound states. This state is expressed with the ansatz

$$
|\psi\rangle=\sum_{n} \int d E c_{n}(E)\left|B_{E}\right\rangle_{n} .
$$

We later show that this is the expected form of the state for a high-power coherent input with a large spectral width. Unlike the previous sections where we select an input pulse and propagate it through the medium, here we simply consider a linear combination of bound eigenstates and compute the observables for this state. A localized function $c_{n}(E)$ ensures that the bound-state ansatz is localized in the center-of-mass coordinate.

Such a state can be probed by measuring either the field $\langle\psi|\hat{a}(x)| \psi\rangle$ or the $m$ th normally ordered observable, which we consider in the center-of-mass coordinate $\left\langle\psi\left|\left[\hat{a}^{\dagger}(x)\right]^{m}[\hat{a}(x)]^{m}\right| \psi\right\rangle$. We compute these observables in the limit where the average photon number is large $\bar{n} \gg 1$, the pulses are spectrally broad $\sigma \ll 1 / \Gamma$, and the order of the correlation function is much less than the photon number $m \ll n$. Within these limits (see the Supplemental Material [30] for full calculations),

$$
\begin{aligned}
\langle\psi|\hat{a}(x)| \psi\rangle & =\frac{\bar{n} \sqrt{\Gamma}}{2} \operatorname{sech}\left(\frac{\bar{n} \Gamma x}{2}\right), \\
\left\langle\psi\left|\left(\hat{a}(x)^{\dagger}\right)^{m}[\hat{a}(x)]^{m}\right| \psi\right\rangle & =\left[\frac{\bar{n} \sqrt{\Gamma}}{2} \operatorname{sech}\left(\frac{\bar{n} \Gamma x}{2}\right)\right]^{2 m},
\end{aligned}
$$

where $\bar{n}$ is the average number of photons in the pulse. These observables reproduce the fundamental soliton solution of SIT [34,35,44] given in Eq. (6). Self-induced transparency is thus the classical limit of the photon bound state when the photon number becomes large, or conversely, photon bound states are simply the quantum limit of a soliton, a quantum soliton. Just like the SIT solitons, the integrated Rabi frequency of the pulse is $\Omega=2 \sqrt{\Gamma} \int d x\langle\hat{a}(x)\rangle=2 \pi$; i.e., the intense pulse of light rapidly excites and deexcites the emitters resulting in a $2 \pi$ Rabi oscillation. We note that, the expressions in Eqs. (17) and (18) scale with $\Gamma$, which is the coupling to the onedimensional continuum. These expressions are therefore unchanged in the presence of coupling to an external reservoir. This implies that, provided the ensemble does 

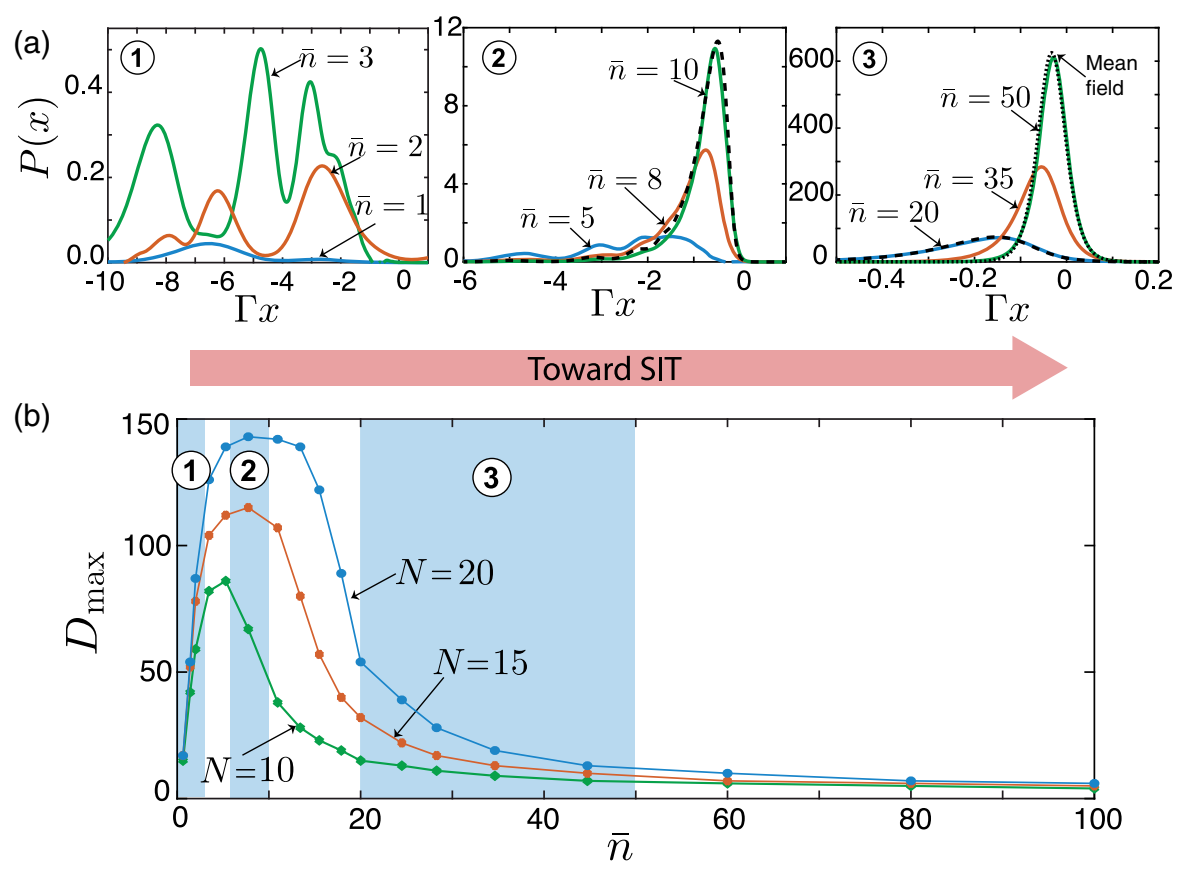

FIG. 3. (a) Output power for $N=20$ atoms for coherent input pulses with a solitonic shape (see text) and different mean photon number $\bar{n}$. The results are computed by directly solving the ME (3) (solid lines) with the MPS algorithm where we fix $D_{\max }=150$. In the second panel, we include the results given in the many-body limit (19) (dashed lines), and in the third the mean-field solitonic ansatz (dotted line). We note that the $x$ axis is limited to focus on larger photon-number components. (b) Maximum bond dimensions $D_{\text {max }}$ of the MPS calculations required to obtain a tolerance less than $10^{-4}$ versus input power $\bar{n}$ for different TLS number $N$. The blue shading highlights the regions for which panels (1)-(3) in (a) show the output power.

not act like a Bragg mirror, SIT is expected to occur even in the presence of backscattering in accordance with meanfield results.

\section{A. Beyond mean-field theory}

As a lowest-order approximation, the variation in photon number $n$ making up the pulse (16) can be ignored, and the state will simply propagate at a reduced speed dictated by the mean photon number. In Eqs. (17) and (18), this lowestorder approximation for the SIT soliton simply maps $x \rightarrow x+4 N /\left(\bar{n}^{2} \Gamma\right)$. However, for a coherent state, the uncertainty in the photon number scales as $\Delta n \sim \sqrt{\bar{n}}$, which leads to a gradual broadening of the pulse due to the different photon-number components accumulating different time delays. This difference in time delay can become significant for a sufficiently large number of emitters $N$. This broadening is not captured by the mean-field theory. The breakdown of the mean-field theory therefore occurs when the difference in the delay of the $\bar{n}$ and the $\bar{n}+\Delta n$ photon bound states becomes on the order of the width of the $\bar{n}$-photon bound state. The ratio of the difference in delay and the width of the $\bar{n}$-photon bound state is given by $\left(\mathcal{T}_{\bar{n}+\Delta n}-\mathcal{T}_{n}\right) / \Delta t_{n} \sim N / n^{3 / 2}$. This means that when $N / n^{3 / 2} \gtrsim 1$ the mean-field theory breaks down even for large input photon number $\bar{n}$. This inequality provides the boundary between mean-field theory and genuine quantum many-body dynamics.
When $\bar{n} \gg 1$ and $N \gtrsim n^{3 / 2}$, one can consider a wave function composed of bound states with a number distribution given by a coherent state. In the limit where meanfield theory breaks down, one must explicitly consider the Fock-state-dependent delay. In this limit, the expression for the field and the power give (see the Supplemental Material [30]),

$$
\begin{aligned}
\langle\hat{a}(x)\rangle & =e^{-|\alpha|^{2}} \sum_{n} \frac{\alpha^{2 n}}{n !} \frac{n \sqrt{\Gamma}}{2} \operatorname{sech}\left[\frac{n \Gamma}{2}\left(x+\frac{4 N}{n^{2} \Gamma}\right)\right], \\
\left\langle\hat{a}^{\dagger}(x) \hat{a}(x)\right\rangle & =e^{-|\alpha|^{2}} \sum_{n} \frac{\alpha^{2 n}}{n !} \frac{n^{2} \Gamma}{4} \operatorname{sech}\left[\frac{n \Gamma}{2}\left(x+\frac{4 N}{n^{2} \Gamma}\right)\right]^{2},
\end{aligned}
$$

with equivalent expressions for higher-order correlation functions. Here, $\alpha=\sqrt{\bar{n}}$ is the coherent field amplitude, which is assumed real. We note that similar expressions exist for the bound state of the nonlinear Schrödinger equation with an attractive interaction $[45,46]$.

Equations (19) provide a simple description of the observables of a quantum many-body state of light. In order to investigate the full transition from the multiphoton bound-state propagation to the formation of the SIT solitons, we make use again of the master equation simulation. In Fig. 3(a), we plot the transmitted power 
of input pulses with $\mathcal{E}_{\text {in }}(x)=(\bar{n} \sqrt{\Gamma} / 2) \operatorname{sech}(\bar{n} \Gamma x / 2)$ for different amplitude strength $\bar{n}$. This pulse shape is chosen such that its electric field matches the SIT criterion. In the first box, we see again the formation of the bound-state peaks which tend to reduce their time delay as the input power is increased. For intermediate input pulses (second box), the bound states with a large number of photons get more populated, and they accumulate toward a single peak as the difference in delay times for large photon numbers becomes less distinguishable. In this regime, the transmitted power starts to be well described by Eq. (19). For even higher input power (third box), the individual bound states are no longer recognizable and a solitonic pulse well described by mean-field theory emerges. These results show how the SIT solitons emerge from a superposition of photon bound states that, in the limit of few photons, can be indeed interpreted as quantum solitons. On the other hand, it is important to emphasize the difference in physical effects. While the SIT solitons can be fully described by a mean-field semiclassical treatment, the formation of distinct bound-state peaks is characterized by a highly correlated state of light and represents the breakdown of the mean-field solution due to quantum effects.

Within the MPS ansatz, one natural way to characterize the amount of correlations in the system is to allow the maximum truncated bond dimension $D_{\max }$ to vary, and to record the value $D_{\max }^{\text {th }}(N, \bar{n})$ at which the truncation error exceeds some acceptable threshold value (see the Supplemental Material [30]). In Fig. 3(b), we show this quantity as a function of the solitonic input pulse strength $\bar{n}$. We see that the breakdown of the mean-field description occurs in the regime where bound-state formation occurs and the amount of correlation in the system is high (large values of $D_{\max }$ ), while in the limit of large $\bar{n}$, the bond dimension tends to shrink approaching the mean-field $\operatorname{limit}\left(D_{\max }^{\mathrm{th}}=1\right)$.

\section{SOLITON INTERACTIONS}

So far, we have characterized the propagation of light through ideal media and shown that it can be understood in terms of the photon bound states. To fully characterize and understand these objects, it is important to also investigate their interactions and robustness to disturbances. To this end, we now make a preliminary investigation of a scattering experiment between a single photon and a two-photon bound state. We consider the input state $\mid$ in $\rangle=$ $C \int d^{3} \mathbf{x a}^{\dagger}(\mathbf{x})|0\rangle \phi\left(x_{1}, x_{2}, x_{3}\right)$ with $C$ being a constant and

$$
\begin{aligned}
\phi\left(x_{1}, x_{2}, x_{3}\right)= & e^{-\left(x_{1}-a_{1}\right)^{2} /\left(2 \sigma^{2}\right)} e^{-\left(x_{2}+x_{3}-a_{2}\right)^{2} /\left(4 \sigma^{2}\right)} \\
& \times e^{-\Gamma / 2\left|x_{3}-x_{2}\right|}+\leftrightarrow
\end{aligned}
$$

i.e., a product state composed of a two-photon bound state and a single photon which are centered at $a_{2}$ and $a_{1}$, respectively, with $a_{2}<a_{1}$. Figure 4 shows the evolution of
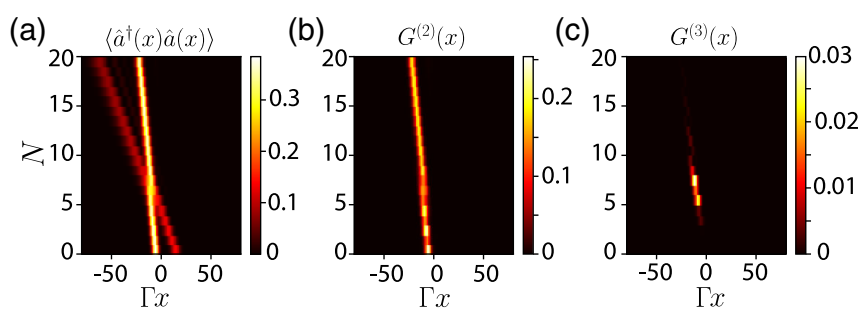

FIG. 4. Evolution of the state $\mid$ in $\rangle$ (see text) demonstrating the interaction between a two-photon bound state centered at $a_{2}=-10 / \Gamma$ and a single photon centered at $a_{1}=15 / \Gamma$ both with width $\sigma \Gamma=3 \sqrt{2}$. The output observables (a) $\left\langle\hat{a}^{\dagger}(x) \hat{a}(x)\right\rangle$, (b) $G^{(2)}(x)$, and (c) $G^{(3)}(x)$ are plotted versus the number of emitters $N$.

the pulse delay for this state as it propagates through the ensemble, i.e., for different $N$. As in previous figures, a frame comoving with the pulse in the absence of interactions (i.e., $N=0$ ) is assumed. Here, since $a_{2}<a_{1}$ and the Wigner delay is larger for single photons $\tau_{1}>\tau_{2}$, the bound-state photons catch up to and overtake the single photon. In this process, the photons interact when the two parts overlap. The interaction causes a change in the Wigner delays, which is seen as kinks in the lines in Figs. 4(a) and 4(b). The region of interaction is highlighted by the third-order correlations shown in Fig. 4(c). After the interaction has ended $(N \gtrsim 10)$, the lines in Fig. 4(a) continue with the same slopes as before the interaction, signifying that there is still a two-photon bound state and an unbound photon. The collision between the bound state and the free photon is thus elastic and the bound state is stable against external influence.

\section{OUTLOOK}

Our theoretical and numerical predictions show that many-body photon bound-state propagation can be observed in chiral waveguide QED geometries with many emitters and photons. While these predictions are in the realm of quantum many-body physics, our work also predicts novel photon transport in the few-photon-fewemitter landscape. This is exemplified in Fig. 5, where we consider coherent pulse propagation with average photon number $\bar{n}=0.5$ and $N=2$ emitters. Figures 5(a) and 5(b) show the output power and correlation functions in the limit of ideal chiral coupling and no loss. Figure 5(a) shows the two-time correlation function $G^{(2)}\left(x_{1}, x_{2}\right)$ for an input pulse with width $\Gamma \sigma=3 \sqrt{2}$. The width of this input pulse is chosen such that, in the two-excitation subspace, it projects on both the two-photon bound-state subspace and the extended states with roughly equal probability. The distinct signatures of these two states can be seen: The bound state clearly propagates faster and is seen as an antinode on the diagonal marked by the intersection of the dashed lines. The spread-out tails which propagate slower 

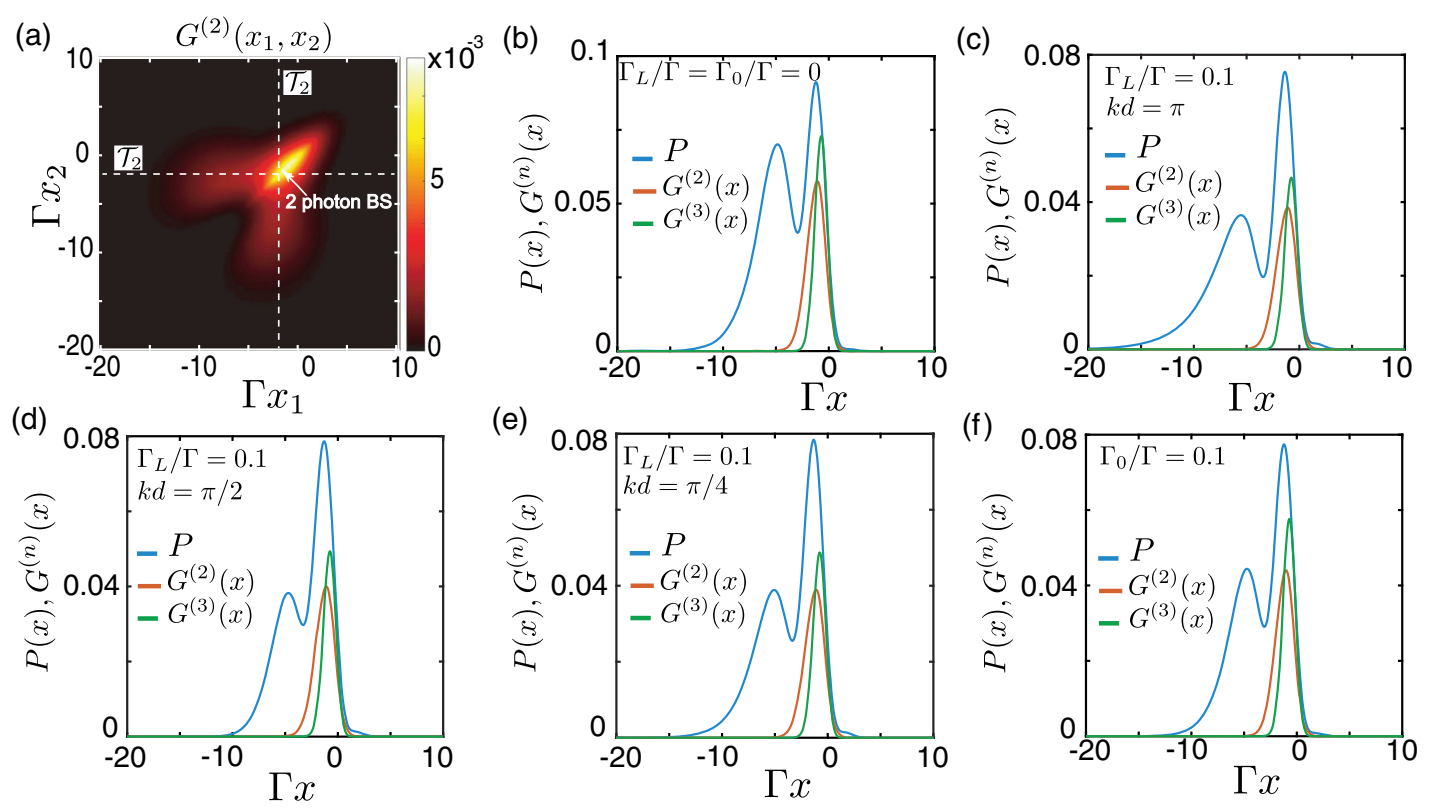

FIG. 5. Propagation of a coherent pulse with average photon number $\bar{n}=0.5$ through $N=2$ emitters. (a) Two-time correlation function $G^{(2)}\left(x_{1}, x_{2}\right)$ for pulse width $\Gamma \sigma=3 \sqrt{2}$ for ideal chiral coupling computed using the three-photon theory. Dashed lines show $N$ times the Wigner delay of the two-photon bound state $\tau_{2}$. (b) Power $P(x)$ and second- and third-order correlation functions $G^{(2)}(x)$ and $G^{(3)}(x)$ for pulse width $\Gamma \sigma=\sqrt{2}$ with ideal coupling. Power and correlations when also coupling to a backward mode with rate $\Gamma_{L}=0.1 \Gamma$ where the emitters are separated by (c) $k d=\pi$, (d) $k d=\pi / 2$, and (e) $k d=\pi / 4$. (f) Power and correlation functions for unidirectional coupling, but with each emitter also coupled to a loss mode with $\Gamma_{0}=0.1 \Gamma$. Panels (b)-(f) are computed using a full numerical treatment.

are the signature of the extended states. These tails are clearly not bunched in comparison to the bound state. Figure 5(b) shows the equal-time correlation function for a narrower pulse width $\Gamma \sigma=\sqrt{2}$. Here, a narrower pulse width is chosen so it dominantly projects on the two-photon bound state. The hallmark of the photon-photon interactions is observed in the difference between the power $P(x)$ and the correlation functions $G^{(2)}(x)$ and $G^{(3)}(x)$. Clearly, the leftmost peak corresponding to the singlephoton component undergoes a larger time delay than the bound states. The difference in time delay between twoand three-photon bound states is also visible in the slight difference between the peak centers of $G^{(2)}(x)$ and $G^{(3)}(x)$. We note that it is also possible to observe a difference between $P(x)$ and $G^{(2)}(x)$ for a single quantum emitter.

We also investigate the robustness of this effect when imperfections are introduced. Figures 5(c)-5(e) consider additional coupling to the left-propagating mode at a rate $\Gamma_{L}=0.1 \Gamma$ for different emitter spacings $k d$, where $k$ is the propagation wave number, and $d$ is the distance between each of the emitters. Note that unlike the fully chiral regime, when $\Gamma_{L} \neq 0$ the distance between emitters influences the dynamics of the system. Although the output field is slightly reduced in all three cases, the difference in the shape of the power and the correlation functions is preserved as is the difference in the peak positions. Finally, Fig. 5(f) considers ideal chiral coupling, but with each emitter coupling to a loss reservoir at a rate $\Gamma_{0}=0.1 \Gamma$.
Here, the single-photon component suffers the largest loss, while the bound states suffer a reduced loss. The introduction of the loss does not spoil the difference in the shape of the power and the correlation functions.

The results here demonstrate that the propagation of fewphoton bound states can be observed with as few as two quantum emitters chirally coupled to a reservoir and is robust to imperfections. This means that the phenomena we show here can be realized by several platforms currently under investigation. Optical quantum dots have demonstrated chiral coupling between a single emitter and a waveguide [47,48], while coupling between two diamond impurity centers and a photonic nanostructure has been achieved [49]. At microwave frequencies, circuit QED platforms can also achieve strong coupling between a superconducting qubit and a transmission line [8] and a scheme for unidirectional coupling has been proposed [50]. Multiple qubits have also been coupled to a single mode or propagating modes [9,51-54]. Finally, gasses of Rydberg atoms under the conditions of electromagnetically induced transparency also exhibit strong nonlinearities [14] and possess bound eigenstates [55]. Under certain limits they can also be mapped onto a nonlinear Schrödinger equation with an attractive interaction [18]. Such a Hamiltonian possesses bound eigenstates [37,46], and therefore, sufficiently long samples of Rydberg gasses can also potentially exhibit the bound-state propagation shown here. Alternatively, ensembles can be engineered 
using Rydberg blockade to mimic chirally coupled emitters [20]. The effects we predict here are thus widely applicable and can be observed in many different physical systems spanning vastly different energy scales.

Throughout this manuscript, we focus on understanding the fundamental physics of photonic bound-state propagation. In addition to its fundamental interest, the dynamics of this system is highly interesting from an applied perspective. As a particular example, we note that, for the output state shown in Figs. 2(a)-2(d), if one selects a temporal window centered at $\Gamma x_{0}=-N$, a state most likely containing either zero or two photons is produced. Launching the output on a beam splitter and conditioning on the detection of a photon will thus produce a single-photon Fock state. For a small-amplitude initial coherent state, the main contribution to this process will arise from the twophoton component of the incoming field. Since the output is in a pure state, the outgoing single photon will also be in a pure state, although the temporal mode function will depend on the detection time. A detailed investigation of using photon bound-state propagation as a photon source is beyond the scope of this work. We can however estimate that for the parameters in Figs. 2(a)-2(d), which are in no way optimized for the application, choosing a window with width $\Gamma x_{w}=8$ centered on $\Gamma x_{0}=-N$ leads to a probability of obtaining two photons $P_{2}=0.061$ and an error probability of obtaining one or three photons $P_{1}+P_{3}=$ $1.5 \times 10^{-3}$. The efficiency and error of the source appear promising as they should be compared with those of the input coherent state which are $P_{2}=0.076$ and $P_{1}+P_{3}=0.32$, respectively.

\section{CONCLUSION}

Our results show that chiral WQED platforms provide a highly nonlinear medium suited for exploring nonlinear optics at the quantum level. From the most elementary lightmatter interaction - the interaction between photons and two-level systems-emerges correlated photonic states. These bound states are truly distinct physical objects with their own dispersion relation and are stable against external influence. Our work provides a clear recipe for how these features can potentially be observed in experiments. In the limit where the number of photons is high, the bound states approach the well-known soliton solution of SIT. Our full quantum description, on the other hand, covers the entire spectrum from few-photon quantum propagation to genuine quantum many-body (atom and photon) phenomena, and ultimately the quantum-to-classical transition. In particular, the analysis highlights how the mean-field solution with weak quantum correlations breaks down through a region of maximal quantum correlations, until finally resulting in a state with weaker correlations. This work therefore paves the way for observing many-body quantum states of light in waveguide QED.

\section{ACKNOWLEDGMENTS}

The authors thank Mantas Čepulkovskis, Peter Lodahl, Sebastian Hofferberth, Hanna le Jeannic, Johannes Bjerlin, L. Henriet, and J. Douglas for fruitful discussions. S. M. and K.H. acknowledge funding from DFG through Grant No. CRC 1227 DQ-mat, Projects No. A05 and A06, and "Niedersächsisches Vorab" through the "Quantum-and Nano-Metrology." G. C. and D. E. C. acknowledge support from ERC Starting Grant FOQAL, MINECO Severo Ochoa Grant No. SEV-2015-0522, CERCA Programme/Generalitat de Catalunya, Fundació Privada Cellex, Fundació Mir-Puig, Fundación Ramón Areces Project CODEC, European Quantum Flagship Project QIA, QuantumCAT (funded within the framework of the ERDF Operational Program of Catalonia), and Plan Nacional Grant ALIQS, funded by Ministerio de Ciencia, Innovación, y Universidades (MCIU), Agencia Estatal de Investigación (AEI), and Fondo Europeo de Desarrollo Regional (FEDER). A. S. S. acknowledges support from the Danish National Research Foundation (Center of Excellence Hy-Q).

[1] D. E. Chang, V. Vuletić, and M. D. Lukin, Quantum Nonlinear Optics_-Photon by Photon, Nat. Photonics 8, 685 (2014).

[2] V. Paulisch, M. Perarnau-Llobet, A. González-Tudela, and J. I. Cirac, Quantum Metrology with One-Dimensional Superradiant Photonic States, Phys. Rev. A 99, 043807 (2019).

[3] D. E. Chang, J. S. Douglas, A. González-Tudela, C.-L. Hung, and H.J. Kimble, Colloquium: Quantum Matter Built from Nanoscopic Lattices of Atoms and Photons, Rev. Mod. Phys. 90, 031002 (2018).

[4] M. Arcari, I. Söllner, A. Javadi, S. L. Hansen, S. Mahmoodian, J. Liu, H. Thyrrestrup, E. H. Lee, J. D. Song, S. Stobbe, and P. Lodahl, Near-Unity Coupling Efficiency of a Quantum Emitter to a Photonic Crystal Waveguide, Phys. Rev. Lett. 113, 093603 (2014).

[5] A. Goban, C.-L. Hung, S.-P. Yu, J. D. Hood, J. A. Muniz, J. H. Lee, M. J. Martin, A. C. McClung, K. S. Choi, D. E. Chang, O. Painter, and H. J. Kimble, Atom-Light Interactions in Photonic Crystals, Nat. Commun. 5, 3808 (2014).

[6] P. Lodahl, S. Mahmoodian, and S. Stobbe, Interfacing Single Photons and Single Quantum Dots with Photonic Nanostructures, Rev. Mod. Phys. 87, 347 (2015).

[7] L. S. Bishop, J. M. Chow, J. Koch, A. A. Houck, M. H. Devoret, E. Thuneberg, S. M. Girvin, and R. J. Schoelkopf, Nonlinear Response of the Vacuum Rabi Resonance, Nat. Phys. 5, 105 (2009).

[8] I.-C. Hoi, T. Palomaki, J. Lindkvist, G. Johansson, P. Delsing, and C. M. Wilson, Generation of Nonclassical Microwave States Using an Artificial Atom in 1D Open Space, Phys. Rev. Lett. 108, 263601 (2012).

[9] M. Mirhosseini, E. Kim, X. Zhang, A. Sipahigil, P. B. Dieterle, A. J. Keller, A. Asenjo-Garcia, D. E. Chang, and O. Painter, Cavity Quantum Electrodynamics with AtomLike Mirrors, Nature (London) 569, 692 (2019). 
[10] K. M. Birnbaum, A. Boca, R. Miller, A. D. Boozer, T. E. Northup, and H. J. Kimble, Photon Blockade in an Optical Cavity with One Trapped Atom, Nature (London) 436, 87 (2005).

[11] A. Faraon, I. Fushman, D. Englund, N. Stoltz, P. Petroff, and J. Vučkovic, Coherent Generation of Non-Classical Light on a Chip via Photon-Induced Tunnelling and Blockade, Nat. Phys. 4, 859 (2008).

[12] A. Reinhard, T. Volz, M. Winger, A. Badolato, K. J. Hennessy, E. L. Hu, and A. Imamoğlu, Strongly Correlated Photons on a Chip, Nat. Photonics 6, 93 (2012).

[13] T. Peyronel, O. Firstenberg, Q.-Y. Liang, S. Hofferberth, A. V. Gorshkov, T. Pohl, M. D. Lukin, and V. Vuletić, Quantum Nonlinear Optics with Single Photons Enabled by Strongly Interacting Atoms, Nature (London) 488, 57 (2012).

[14] O. Firstenberg, T. Peyronel, Q.-Y. Liang, A. V. Gorshkov, M. D. Lukin, and V. Vuletić, Attractive Photons in a Quantum Nonlinear Medium, Nature (London) 502, 71 (2013).

[15] A. Javadi, I. Söllner, M. Arcari, S. L. Hansen, L. Midolo, S. Mahmoodian, G. Kiršanskè, T. Pregnolato, E. H. Lee, J. D. Song, S. Stobbe, and P. Lodahl, Single-Photon Non-Linear Optics with a Quantum Dot in a Waveguide, Nat. Commun. 6, 8655 (2015).

[16] C. Hamsen, K. N. Tolazzi, T. Wilk, and G. Rempe, TwoPhoton Blockade in an Atom-Driven Cavity QED System, Phys. Rev. Lett. 118, 133604 (2017).

[17] L. De Santis, C. Antón, B. Reznychenko, N. Somaschi, G. Coppola, J. Senellart, C. Gómez, A. Lemaître, I. Sagnes, A. G. White, L. Lanco, A. Aufféves, and P. Senellart, A Solid-State Single-Photon Filter, Nat. Nanotechnol. 12, 663 (2017).

[18] Q.-Y. Liang, A. V. Venkatramani, S. H. Cantu, T. L. Nicholson, M. J. Gullans, A. V. Gorshkov, J. D. Thompson, C. Chin, M. D. Lukin, and V. Vuletić, Observation of ThreePhoton Bound States in a Quantum Nonlinear Medium, Science 359, 783 (2018).

[19] S. Mahmoodian, M. Čepulkovskis, S. Das, P. Lodahl, K. Hammerer, and A. S. Sørensen, Strongly Correlated Photon Transport in Waveguide Quantum Electrodynamics with Weakly Coupled Emitters, Phys. Rev. Lett. 121, 143601 (2018).

[20] N. Stiesdal, J. Kumlin, K. Kleinbeck, P. Lunt, C. Braun, A. Paris-Mandoki, C. Tresp, H. P. Büchler, and S. Hofferberth, Observation of Three-Body Correlations for Photons Coupled to a Rydberg Superatom, Phys. Rev. Lett. 121, 103601 (2018).

[21] E. P. Wigner, Lower Limit for the Energy Derivative of the Scattering Phase Shift, Phys. Rev. 98, 145 (1955).

[22] R. Bourgain, J. Pellegrino, S. Jennewein, Y. R. P. Sortais, and A. Browaeys, Direct Measurement of the Wigner Time Delay for the Scattering of Light by a Single Atom, Opt. Lett. 38, 1963 (2013).

[23] G. Nikoghosyan and M. Fleischhauer, Photon-Number Selective Group Delay in Cavity Induced Transparency, Phys. Rev. Lett. 105, 013601 (2010).

[24] H. Tanji-Suzuki, W. Chen, R. Landig, J. Simon, and Vuletić, Vacuum-Induced Transparency, Science 333, 1266 (2011).
[25] P. Lodahl, S. Mahmoodian, S. Stobbe, A. Rauschenbeutel, P. Schneeweiss, J. Volz, H. Pichler, and P. Zoller, Chiral Quantum Optics, Nature (London) 541, 473 (2017).

[26] T. Caneva, M. T. Manzoni, T. Shi, J. S. Douglas, J. I. Cirac, and D.E. Chang, Quantum Dynamics of Propagating Photons with Strong Interactions: A Generalized InputOutput Formalism, New J. Phys. 17, 113001 (2015).

[27] M. T. Manzoni, D. E. Chang, and J. S. Douglas, Simulating Quantum Light Propagation through Atomic Ensembles Using Matrix Product States, Nat. Commun. 8, 1743 (2017).

[28] K. Stannigel, P. Rabl, and P. Zoller, Driven-Dissipative Preparation of Entangled States in Cascaded QuantumOptical Networks, New J. Phys. 14, 063014 (2012).

[29] H. Pichler, T. Ramos, A. J. Daley, and P. Zoller, Quantum Optics of Chiral Spin Networks, Phys. Rev. A 91, 042116 (2015).

[30] See Supplemental Material at http://link.aps.org/ supplemental/10.1103/PhysRevX.10.031011 for further details of the calculations.

[31] F. Verstraete, V. Murg, and J. I. Cirac, Matrix Product States, Projected Entangled Pair States, and Variational Renormalization Group Methods for Quantum Spin Systems, Adv. Phys. 57, 143 (2008).

[32] U. Schollwöck, The Density-Matrix Renormalization Group in the Age of Matrix Product States, Ann. Phys. (Amsterdam) 326, 96 (2011).

[33] S. L. McCall and E. L. Hahn, Self-Induced Transparency by Pulsed Coherent Light, Phys. Rev. Lett. 18, 908 (1967).

[34] S. L. McCall and E. L. Hahn, Self-Induced Transparency, Phys. Rev. 183, 457 (1969).

[35] R. K. Bullough, P. J. Caudrey, J. C. Eilbeck, and J. D. Gibbon, A General Theory of Self-Induced Transparency, Opto-electronics 6, 121 (1974).

[36] H. A. Bethe, On the Theory of Metals, Z. Phys. 71, 205 (1931).

[37] H. B. Thacker, Exact Integrability in Quantum Field Theory and Statistical Systems, Rev. Mod. Phys. 53, 253 (1981).

[38] V. I. Rupasov and V. I. Yudson, Exact Dicke Superradiance Theory: Bethe Wavefunctions in the Discrete Atom Model, Zh. Eksp. Teor. Fiz. 86, 819 (1984) [Sov. Phys. JETP 59, 478 (1984)].

[39] J.-T. Shen and S. Fan, Strongly Correlated Multiparticle Transport in One Dimension through a Quantum Impurity, Phys. Rev. A 76, 062709 (2007).

[40] H. Zheng, D. J. Gauthier, and H. U. Baranger, Cavity-Free Photon Blockade Induced by Many-Body Bound States, Phys. Rev. Lett. 107, 223601 (2011).

[41] Y. Shen and J.-T. Shen, Photonic-Fock-State Scattering in a Waveguide-QED System and Their Correlation Functions, Phys. Rev. A 92, 033803 (2015).

[42] V. I. Yudson, Dynamics of Integrable Quantum Systems, Zh. Eksp. Teor. Fiz. 88, 1757 (1985) [Sov. Phys. JETP 88, 1043 (1985)].

[43] P. Bienias, J. Douglas, A. Paris-Mandoki, P. Titum, I. Mirgorodskiy, C. Tresp, E. Zeuthen, M. J. Gullans, M. Manzoni, S. Hofferberth, D. Chang, and A. Gorshkov, Photon Propagation through Dissipative Rydberg Media 
at Large Input Rates, arXiv:1807.07586 [Phys. Rev. X (to be published)].

[44] A. V. Yulin, I. V. Iorsh, and I. A. Shelykh, Resonant Interaction of Slow Light Solitons and Dispersive Waves in Nonlinear Chiral Photonic Waveguide, New J. Phys. 20, 053065 (2018).

[45] Y. Lai and H. A. Haus, Quantum Theory of Solitons in Optical Fibers. I. Time-Dependent Hartree Approximation, Phys. Rev. A 40, 844 (1989).

[46] Y. Lai and H. A. Haus, Quantum Theory of Solitons in Optical Fibers. II. Exact Solution, Phys. Rev. A 40, 854 (1989).

[47] I. Söllner, S. Mahmoodian, S. L. Hansen, L. Midolo, G. Kirsanske, T. Pregnolato, H. El-Ella, E. H. Lee, J. D. Song, S. Stobbe, and P. Lodahl, Deterministic Photon-Emitter Coupling in Chiral Photonic Circuits, Nat. Nanotechnol. 10, 775 (2015).

[48] A. Javadi, D. Ding, M. H. Appel, S. Mahmoodian, M. C. Löbl, I. Söllner, R. Schott, C. Papon, T. Pregnolato, S. Stobbe, L. Midolo, T. Schröder, A. D. Wieck, A. Ludwig, R. J. Warburton, and P. Lodahl, Spin-Photon Interface and Spin-Controlled Photon Switching in a Nanobeam Waveguide, Nat. Nanotechnol. 13, 398 (2018).

[49] A. Sipahigil, R. E. Evans, D. D. Sukachev, M. J. Burek, J. Borregaard, M. K. Bhaskar, C. T. Nguyen, J. L. Pacheco, H. A. Atikian, C. Meuwly, R. M. Camacho, F. Jelezko, E. Bielejec, H. Park, M. Lončar, and M. D. Lukin, An
Integrated Diamond Nanophotonics Platform for QuantumOptical Networks, Science 354, 847 (2016).

[50] P. O. Guimond, B. Vermersch, M. L. Juan, A. Sharafiev, G. Kirchmair, and P. Zoller, A Unidirectional On-Chip Photonic Interface for Superconducting Circuits, npj Quantum Inf. 6, 32 (2020).

[51] A. F. Van Loo, A. Fedorov, K. Lalumière, B. C. Sanders, A. Blais, and A. Wallraff, Photon-Mediated Interactions between Distant Artificial Atoms, Science 342, 1494 (2013).

[52] P. Y. Wen, K.-T. Lin, A. Kockum, B. Suri, H. Ian, J. C. Chen, S. Y. Mao, C. C. Chiu, P. Delsing, F. Nori, G.-D. Lin, and I.-C. Hoi, Large Collective Lamb Shift of Two Distant Superconducting Artificial Atoms, Phys. Rev. Lett. 123, 233602 (2019).

[53] M. Fitzpatrick, N. M. Sundaresan, A. C. Y. Li, J. Koch, and A. A. Houck, Observation of a Dissipative Phase Transition in a One-Dimensional Circuit QED Lattice, Phys. Rev. X 7, 011016 (2017).

[54] N. M. Sundaresan, R. Lundgren, G. Zhu, A. V. Gorshkov, and A. A. Houck, Interacting Qubit-Photon Bound States with Superconducting Circuits, Phys. Rev. X 9, 011021 (2019).

[55] M. F. Maghrebi, M. J. Gullans, P. Bienias, S. Choi, I. Martin, O. Firstenberg, M. D. Lukin, H. P. Büchler, and A. V. Gorshkov, Coulomb Bound States of Strongly Interacting Photons, Phys. Rev. Lett. 115, 123601 (2015). 